\title{
$511 \mathrm{keV} \gamma$-ray emission from the galactic bulge by $\mathrm{MeV}$ millicharged dark matter ${ }^{1}$
}

\author{
Ji-Haeng Huh, Jihn E. Kim, Jong-Chul Park, and Seong Chan Park \\ Department of Physics and Astronomy, Seoul National University, Seoul 151-747, Korea
}

\begin{abstract}
We propose a possible explanation for the recently observed anomalous $511 \mathrm{keV}$ line with a new "millicharged" fermion. This new fermion is light $[\mathscr{O}(\mathrm{MeV})]$. Nevertheless, it has never been observed by any collider experiments by virtue of its tiny electromagnetic charge $\varepsilon e$. In particular, we constrain parameters of this millicharged particle if the $511 \mathrm{keV}$ cosmic $\gamma$-ray emission from the galactic bulge is due to positron production from this new particle.
\end{abstract}

Keywords: Millicharged particles, $511 \mathrm{keV}$ line, Dark matter, Galactic bulge PACS: $95.35 .+\mathrm{d}, 12.60 . \mathrm{Cn}, 98.70 . \mathrm{Rz}$

\section{INTRODUCTION}

After the first detection of $\sim 500 \mathrm{keV} \gamma$-rays from the galactic center (GC) [1], various observations have been performed. The recent SPI/INTEGRAL observation shows the very sharp peak from the galactic bulge, which is very well consistent with the electron mass $m_{e} \simeq 511 \mathrm{keV}$ [2, 3]. The morphology of the emission region is consistent with a 2-dimensional gaussian of a full width at half maximum of $6^{\circ}$ with a $2 \sigma$ uncertainty range, $4^{\circ}-9^{\circ}$. The most probable explanation of this line emission is that it comes from the positronium decay. Therefore, a sufficient number of positrons are needed. Some obvious candidate origins for positrons are the astrophysical sources such as massive stars, hypernovae, cosmic-ray interactions, X-ray binaries, type Ia supernovae. However, these sources are inappropriate to explain the intensity of $511 \mathrm{keV} \gamma$-ray flux and the shape of emission region. Thus, particle physics origins with new particles are currently more preferred. One of them is low mass $(\sim \mathrm{MeV})$ particle dark matter $(\mathrm{DM})$ annihilation [4]. Along this idea, we propose a new DM candidate with $\mathscr{O}(\mathrm{MeV})$ mass and very small electromagnetic charge 3 which can explain the galactic $511 \mathrm{keV} \gamma$-rays.

If there exists another massless $U(1)$ gauge boson, "exphoton," beyond the standard model (SM), most probably a kinetic mixing is generated via loop effects between the photon and exphoton. After a proper diagonalization procedure of kinetic energy terms, hidden sector particles can be electromagnetically millicharged $[5,3$

\footnotetext{
${ }^{1}$ A talk given by J. Park at SUSY'08 (Seoul, Korea, June 16-21, 2008).

2 Only neutral particles are typically considered as DM candidates. However, charged particles also could be a good candidate if their electric charge is sufficiently tiny.

3 The term "millicharged" does not mean really a milli-size electromagnetic charge but just a small charge.
}

Laboratory and cosmological bounds of millicharged particles are well summarized in Ref. [6], but some constraints such as the $511 \mathrm{keV}$ photon flux and the Debye screening are not included. Therefore, we investigate the possibility of the $\mathscr{O}(\mathrm{MeV})$ millicharged particles toward interpreting the $511 \mathrm{keV}$ line emission and some related constraints [7].

\section{KINETIC MIXING AND MILLICHARGED PARTICLE}

For $U(1)_{\mathrm{em}}$ and $U(1)_{\mathrm{ex}}$ symmetries, the kinetic mixing of $U(1)_{\mathrm{em}}$ and $U(1)_{\mathrm{ex}}$ gauge bosons is parameterized as

$$
\mathscr{L}=-\frac{1}{4} \hat{F}_{\mu v} \hat{F}^{\mu v}-\frac{1}{4} \hat{X}_{\mu v} \hat{X}^{\mu v}-\frac{\xi}{2} \hat{F}_{\mu v} \hat{X}^{\mu v},
$$

where $\hat{A}_{\mu}$ and $\hat{X}_{\mu}$ are $U(1)_{\mathrm{em}}$ and $U(1)_{\mathrm{ex}}$ gauge bosons, and their field strength tensors are $\hat{F}_{\mu \nu}$ and $\hat{X}_{\mu \nu}$ respectively. Although the kinetic mixing parameter $\xi$ is expected to be generated by an ultraviolet theory [5], $\xi$ can be treated as a arbitrary parameter in a low energy effective theory. A proper transformation of the gauge fields,

$$
\left(\begin{array}{c}
A_{\mu} \\
X_{\mu}
\end{array}\right)=\left(\begin{array}{cc}
\sqrt{1-\xi^{2}} & 0 \\
\xi & 1
\end{array}\right)\left(\begin{array}{c}
\hat{A}_{\mu} \\
\hat{X}_{\mu}
\end{array}\right)
$$

leads to

$$
\mathscr{L}=-\frac{1}{4} F_{\mu v} F^{\mu v}-\frac{1}{4} X_{\mu v} X^{\mu v},
$$

where photon and exphoton correspond to $A_{\mu}$ and $X_{\mu}$ respectively, and $F_{\mu \nu}$ and $X_{\mu \nu}$ are the new field strengths.

Let us take the following interaction Lagrangian of a SM fermion, i.e. electron, in the original basis as

$$
\mathscr{L}=\bar{\psi}\left(\hat{e} Q \gamma^{\mu}\right) \psi \hat{A}_{\mu}
$$


If there is a hidden sector fermion $\chi$ with a $U(1)_{\text {ex }}$ charge $Q_{\chi}$, its interaction with the hidden gauge boson is given by

$$
\mathscr{L}=\bar{\chi}\left(\hat{e}_{\mathrm{ex}} Q_{\chi} \gamma^{\mu}\right) \chi \hat{X}_{\mu}
$$

Note that no direct interaction exists between the electron and the hidden gauge boson $\hat{X}$, and also between the hidden fermion and the visible sector gauge boson $\hat{A}$.

In the transformed basis, Eq. (4) is rewritten as

$$
\mathscr{L}=\bar{\psi}\left(\frac{\hat{e}}{\sqrt{1-\xi^{2}}} Q \gamma^{\mu}\right) \psi A_{\mu} .
$$

Even after the change of basis, the SM fermion has a coupling only to the photon $A$. On the other hand, the coupling $\hat{e}$ is modified to $\hat{e} / \sqrt{1-\xi^{2}}$; consequently, the physical visible sector coupling $e$ is defined as $e \equiv$ $\hat{e} / \sqrt{1-\xi^{2}}$. Similarly, we obtain the following for $\chi$,

$$
\mathscr{L}=\bar{\chi} \gamma^{\mu}\left(\hat{e}_{\mathrm{ex}} Q_{\chi} X_{\mu}-\hat{e}_{\mathrm{ex}} \frac{\xi}{\sqrt{1-\xi^{2}}} Q_{\chi} A_{\mu}\right) \chi .
$$

In this basis, the hidden fermion $\chi$ can couple to the photon $A$ with the coupling $-\hat{e}_{\mathrm{ex}} \xi / \sqrt{1-\xi^{2}}$. Thus, we set the physical hidden coupling as $e_{\mathrm{ex}} \equiv \hat{e}_{\mathrm{ex}}$ and define the coupling of $\chi$ to the photon $A$ as $\varepsilon e \equiv-e_{\mathrm{ex}} \xi / \sqrt{1-\xi^{2}}$ by introducing the millicharge parameter $\varepsilon$. Note that $e \neq e_{\mathrm{ex}}$ in general. In principle, one can calculate the ratio $e_{\mathrm{ex}} / e$ from a fundamental theory. However, we simply take the ratio as a free parameter.

\section{CONSTRAINTS ON LIGHT MILLICHARGED DARK MATTER}

Needed cross sections for the cosmological study of $\chi$ are $\chi \bar{\chi} \rightarrow 2 \gamma_{\mathrm{ex}}, \chi \bar{\chi} \rightarrow e^{-} e^{+}, \chi \bar{\chi} \rightarrow \gamma \gamma_{\mathrm{ex}}$, and $\chi \bar{\chi} \rightarrow \gamma \gamma$. The ratio for these cross sections is given by

$$
\sigma_{2 \gamma_{\mathrm{ex}}}: \sigma_{e+e-}: \sigma_{\gamma \gamma_{\mathrm{ex}}}: \sigma_{2 \gamma} \simeq \alpha_{\mathrm{ex}}^{2}: \varepsilon^{2} \alpha^{2}: \varepsilon^{2} \alpha \alpha_{\mathrm{ex}}: \varepsilon^{4} \alpha^{2} \text {. }
$$

The first two channels mainly determine the relic density of $\chi$ since the last two channels are quite suppressed in the parameter region where $\varepsilon$ and $\alpha_{\mathrm{ex}} / \alpha$ are small as is required by observational data. If $\alpha_{\mathrm{ex}} / \alpha>0.01(0.1)$, the background diffuse $\gamma$-ray flux could be larger than $1(10) \%$ of the $511 \mathrm{keV}$ flux, so the region is already excluded [8, 9] (see the upper (green) region in Fig. 10. The second process determines the $511 \mathrm{keV}$ flux as well.

The relic density of a generic relic, $X$, is given by

$$
\Omega_{X} h^{2} \approx \frac{1.07 \times 10^{9} \mathrm{GeV}^{-1}}{M_{P l}} \frac{x_{F}}{\sqrt{g_{*}}} \frac{1}{\left(a+3 b / x_{F}\right)},
$$

where $g_{*}$ is evaluated at the freeze-out temperature $T_{F}$, $x_{F}=m_{X} / T_{F} \simeq 17.2+\ln \left(g / g_{*}\right)+\ln \left(m_{X} / \mathrm{GeV}\right)+\ln \sqrt{x_{F}}$

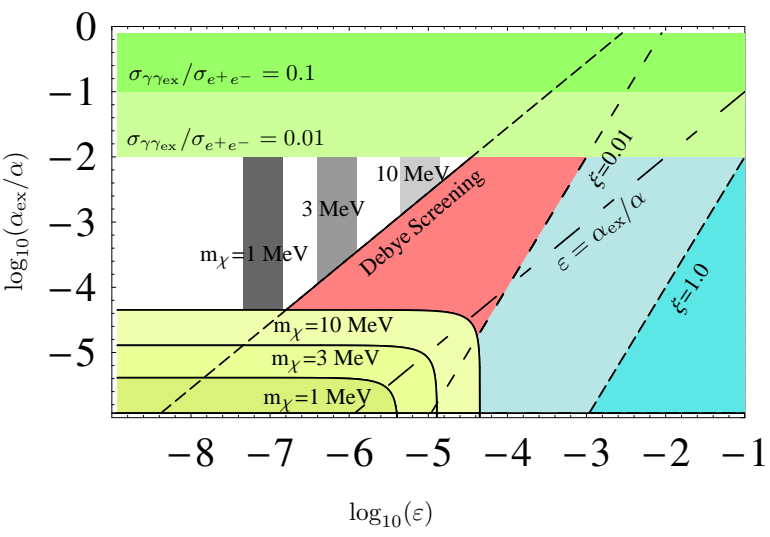

FIGURE 1. The plot for $\alpha_{\mathrm{ex}} / \alpha$ versus $\varepsilon$.

for $1 \mathrm{MeV} \lesssim m_{X} \lesssim 1 \mathrm{GeV}$, and the cosmological average of the cross section times velocity is expressed as $\langle\sigma v\rangle=a+b\left\langle v^{2}\right\rangle+\mathscr{O}\left(\left\langle v^{4}\right\rangle\right)$ [10]. Using $a=a_{e^{-} e^{+}}+a_{2 \gamma_{\mathrm{ex}}}$, $b=b_{e^{-} e^{+}}+b_{2 \gamma_{\mathrm{ex}}}$, and an approximated relation $x_{F} \approx$ $11.6+\ln \left(m_{X} / \mathrm{MeV}\right)$ for $1 \mathrm{MeV} \lesssim m_{X} \lesssim 100 \mathrm{MeV}$, we can estimate the relic density of the millicharged particle $\chi$ as

$$
\Omega_{\chi} h^{2} \approx \frac{1.60 \times 10^{-13}(11.6+\ln \bar{m}) \bar{m}^{2}}{\left(\frac{\alpha_{\mathrm{ex}}}{\alpha}\right)^{2}+\varepsilon^{2}\left(1-\frac{m_{e}^{2}}{m_{\chi}^{2}}\right)^{1 / 2}\left(1+\frac{m_{e}^{2}}{2 m_{\chi}^{2}}\right)},
$$

where $\bar{m} \equiv m_{\chi} / \mathrm{MeV}$ and we put $g_{*} \simeq 10.75$ for $1<$ $T_{F} / \mathrm{MeV}<100$. Finally, we find a constraint for $m_{\chi}$, $\varepsilon$, and $\alpha_{\mathrm{ex}} \equiv e_{\mathrm{ex}}^{2} / 4 \pi$, based on the WMAP three-year results [11]. In Fig. 11, the lower left (yellow) corner is excluded by our DM relic density analysis: the lines correspond to $\Omega_{\chi} h^{2}=0.11$ and $m_{\chi}=1,3$, and $10 \mathrm{MeV}$, respectively. Recent analysis such as internal bremsstrahlung radiation and in-flight annihilation gives strong mass bound for $\mathrm{DM}$ in $\mathrm{MeV}$ region: $m \lesssim 3-4$ $\mathrm{MeV}$ [9]. This bound can be reduced by a factor of two by a possible ionization of the medium [12]. Therefore, (8) in this study we focus on the mass range, $m_{\chi} \lesssim 10 \mathrm{MeV}$.

If $m_{\chi}<m_{\mu}$, the low velocity annihilations dominantly produce $e^{-} e^{+}$pairs. Most positrons lose energy through their interactions with the inter stellar medium and bremsstrahlung radiation, and go rest. Thus, positron annihilation takes place via the positronium formation $(\sim 96.7 \pm 2.2 \%)$ [3] and partly via the direct annihilation into two $511 \mathrm{keV} \gamma$-rays. A singlet positronium state decays to two $511 \mathrm{keV}$ photons $(25 \%)$, whereas a triplet state decays to three continuum photons $(75 \%)$. As a result, the $511 \mathrm{keV} \gamma$-ray flux from the galactic bulge can be given as

$$
\begin{aligned}
\Phi_{\gamma, 511} \simeq & 0.275 \times 5.6 \bar{J}(\Delta \Omega) \Delta \Omega \\
& \times\left(\frac{\sigma v}{\mathrm{pb}}\right)\left(\frac{1 \mathrm{MeV}}{m_{\chi}}\right)^{2} \mathrm{~cm}^{-2} \mathrm{~s}^{-1},
\end{aligned}
$$


where $\Delta \Omega$ is the observed solid angle toward the GC and $\bar{J}(\Delta \Omega)$ is defined as the average of $J(\psi)$ over a spherical region of solid angle $\Delta \Omega$ centered on $\psi=0$ [10]. Here, we separate halo profile depending factors from particle physics depending factors by introducing the quantity $J(\psi)$ :

$$
J(\psi)=\frac{1}{8.5 \mathrm{kpc}}\left(\frac{1}{0.3 \mathrm{GeV} / \mathrm{cm}^{3}}\right)^{2} \int_{1.0 . \mathrm{s}} d s \rho^{2}(r(s, \psi)),
$$

where $s$ is a coordinate running along the line of sight (l.o.s) in a direction making an angle $\psi$ from the direction of the GC.

$\Phi_{\gamma, 511} \simeq(1.02 \pm 0.10) \times 10^{-3} \mathrm{ph} \mathrm{cm}^{-2} \mathrm{~s}^{-1}[2,3]$ and $e^{+}$is produced from the process $\chi \bar{\chi} \rightarrow e^{-} e^{+}$. Therefore, considering a solid angle of $0.0086 \mathrm{sr}$ corresponding to a $6^{\circ}$ diameter circle, we can find the charge $\varepsilon$ of the millicharged DM as a function of its mass $m_{\chi}$ :

$\varepsilon \simeq 1.0 \times 10^{-6} \frac{\bar{m}^{2}}{\sqrt{\bar{J}}}\left[1-\frac{m_{e}^{2}}{m_{\chi}^{2}}\right]^{-1 / 4}\left[1+\frac{m_{e}^{2}}{2 m_{\chi}^{2}}\right]^{-1 / 2}$.

To estimate the required parameter space, we use the width of the observed distribution $\bar{J}(0.0086 \mathrm{sr}) \sim 50-$ 500 , approximately corresponding to $\gamma \simeq 0.6-1.2$, essentially following the approach of Ref. [4], 4 In Fig. 1] we show the allowed range of $\varepsilon$ for typical DM masses $\left(m_{\chi}=1,3\right.$, and $10 \mathrm{MeV}$ ) as the (grey) vertical bands, obtained from the $511 \mathrm{keV} \gamma$-ray flux analysis.

If DM is charged, photon obtains effective mass in the DM plasma, and this mass should be smaller than the experimental limit. As a result, the Debye screening length in the DM plasma around Earth $\lambda_{D}=\sqrt{T_{\chi} / \mathcal{E}^{2} e^{2} n_{\chi}}$ is required to be larger than $1 / m_{\gamma}^{\text {eff }}$ [13]. Putting $n_{\chi}=$ $\rho_{\chi} / m_{\chi} \simeq 0.3 \mathrm{GeV} / \mathrm{cm}^{3} \times \Omega_{\chi} /\left(\Omega_{\mathrm{DM}} m_{\chi}\right)$ and $\Omega_{\mathrm{DM}} \simeq$ 0.23 , we finally obtain a simple relation $\frac{\alpha_{\mathrm{ex}}}{\alpha} \gtrsim 282 \varepsilon$. The lower right corner (pink) from the central region is excluded by this constraint.

In the upper part of the line $\varepsilon=\alpha_{\mathrm{ex}} / \alpha$ the process $\chi \bar{\chi} \rightarrow 2 \gamma_{\text {ex }}$ and in the lower part the process $\chi \bar{\chi} \rightarrow e^{-} e^{+}$ dominates respectively. Therefore, in the allowed parameter region $\left(1 \gg \alpha_{\mathrm{ex}} / \alpha>\varepsilon\right)$, the relic density of DM is essentially determined by $\chi \bar{\chi} \rightarrow 2 \gamma_{\text {ex }}$. However, the observed $511 \mathrm{keV}$ photon flux is mostly explained by $\chi \bar{\chi} \rightarrow e^{-} e^{+}$. In this respect, the difficulty of explaining both quantities in Ref. [4] is easily evaded in our model. As can be seen from Fig. 1, a significant region is excluded. However, we note that there still remains an available space.

\footnotetext{
${ }^{4}$ If the main source of $511 \mathrm{keV} \gamma$-rays from galactic bulge is the DM annihilation, the observed distribution of $511 \mathrm{keV}$ emission line would constrain the shape of the DM halo profile because DM annihilation rate is proportional to the DM density squared.
}

\section{CONCLUSION}

We suggested the $\mathrm{MeV}$ millicharged dark matter as a possible solution for the recently observed anomalous $511 \mathrm{keV}$ cosmic $\gamma$-rays. In this regard, we considered various bounds including the relic density, the Debye screening, and the diffuse $\gamma$-ray background. From this study, we conclude that the millicharged particle hypothesis is not ruled out yet but there remains only a small parameter space compatible with the $511 \mathrm{kev} \gamma$-ray flux. Finally, we note that a millicharged particle with a small mass is preferred as long as its mass is larger than the electron mass $m_{e}$ for it to constitute a sizable portion of the dark matter content of the Universe.

\section{ACKNOWLEDGMENTS}

This work was supported in part by the Korea Research Foundation Grant funded by the Korean Goverment (MOEHRD) (KRF-2005-084-C00001).

\section{REFERENCES}

1. W. N. Johnson, F. R. Harnden and R. C. Haymes, Astrophys. J. 172, L1 (1972).

2. J. Knodlseder et al., Astron. Astrophys. 411, L457 (2003); P. Jean et al., Astron. Astrophys. 407, L55 (2003); J. Knodlseder et al., Astron. Astrophys. 441, 513 (2005); G. Weidenspointner et al., Astron. Astrophys. 450, 1013 (2006).

3. P. Jean et al., Astron. Astrophys. 445, 579 (2006)

4. C. Boehm, D. Hooper, J. Silk, M. Casse and J. Paul, Phys. Rev. Lett. 92, 101301 (2004).

5. L. B. Okun, Zh. Eksp. Teor. Fiz. 83, 892 (1982) [Sov. Phys. JETP 56, 502 (1982)]; B. Holdom, Phys. Lett. B166, 196 (1986).

6. S. Davidson, S. Hannestad and G. Raffelt, JHEP 0005, 003 (2000).

7. J. H. Huh, J. E. Kim, J. C. Park and S. C. Park, Phys. Rev. D 77, 123503 (2008).

8. A. W. Strong, I. V. Moskalenko and O. Reimer, Astrophys. J. 537, 763 (2000) [Erratum-ibid. 541, 1109 (2000)].

9. J. F. Beacom and H. Yuksel, Phys. Rev. Lett. 97, 071102 (2006).

10. G. Bertone, D. Hooper and J. Silk, Phys. Rep. 405, 279 (2005)

11. D. N. Spergel et al. (WMAP Collaboration), Astrophys. J. Suppl. 170, 377 (2007).

12. P. Sizun, M. Casse, S. Schanne and B. Cordier, arXiv:astro-ph/0702061

13. E. R. Williams, J. E. Faller and H. A. Hill, Phys. Rev. Lett. 26, 721 (1971); S. Mitra, Phys. Rev. D 74, 043532 (2006). 